\title{
Hypercalcaemia management in a district general hospital
}

\author{
Authors: Sukanya Ghosh ${ }^{\mathrm{A}}$ and Jude Edward ${ }^{\mathrm{B}}$
}

\section{Introduction}

Hypercalcaemia is a common finding in inpatients. Acute hypercalcaemia can be life-threatening and thus proper workup is pivotal for correct assessment of the underlying cause and management. The European Guidelines $2016^{1}$ were used to assess the investigation and management of patients with hypercalcaemia.

\section{Aim}

We undertook a study to assess the appropriateness in work-up, diagnosis and management of patients with hypercalcaemia.

\section{Materials and methods}

This was a retrospective study. Patients diagnosed with hypercalcaemia between November 2017 and December 2018 admitted to a district general hospital across different medical and surgical specialties were included in the study. Data were obtained from medical records for symptoms of hypercalcaemia, location of patient in the hospital, biochemical and laboratory tests (including adjusted serum calcium (SCa), and data on patients' management were obtained from the case notes.

\section{Results and discussion}

Of the 50 patients included in the study, 17 were male with a mean age of 76.1 years. The majority of patients $(n=43 ; 84 \%)$ had mild hypercalcaemia (SCa 2.67-2.9 mmol/L); moderate hypercalcaemia (SCa $3.0-3.4 \mathrm{mmol} / \mathrm{L}$ ) was found in five patients $(10 \%)$ and severe hypercalcaemia $(\mathrm{SCa} \geq 3.5 \mathrm{mmol} / \mathrm{L})$ in three patients $(6 \%)$. Thirty-one patients $(62 \%)$ demonstrated symptoms of hypercalcaemia. All patients had renal function assessed on admission, phosphate was measured in 98\%, parathyroid hormone (PTH) in 19 (38\%) patients and vitamin $\mathrm{D}$ in $32 \%$. Electrocardiography (ECG) was done in $24 \%$, and one patient had ECG changes. Of the patients who had PTH measured, 52\% had high PTH levels, 31\% had low PTH and $15 \%$ had normal PTH levels. Of those with high/normal PTH, $76 \%$ were referred to an endocrinologist. $16 \%$ of patients had known malignancy. $6 \%$ of patients with low PTH had

Authors: ${ }^{\text {AB }}$ lackpool Victoria Hospital, Blackpool, UK; ${ }^{\mathrm{B}}$ Tameside General Hospital, Ashton-under-Lyne, UK malignancy. Of the patients with malignancy, PTH and vitamin $\mathrm{D}$ were not done in $12 \%$. Management varied within the group: $14 \%$ of patients received intravenous fluids, bisphosphonates were given to $8 \%$, steroids to $8 \%$, and $2 \%$ received dialysis. $74 \%$ had repeat adjusted SCa measured after 24 hours. No patient had 24-hour urine calcium. Management of hypercalcaemia was appropriate in $60 \%$ of patients. Only $12 \%$ had follow-up with endocrinology.

\section{Conclusion}

Guidelines were poorly followed for inpatients in a district general hospital for the assessment and management of hypercalcaemia. Training of healthcare professionals is essential so that guidelines are followed to deliver the best care to patients with hypercalcaemia.

\section{Conflicts of interest}

None declared.

\section{Reference}

1 Walsh J, Gittoes N, Selby P, Society for Endocrinology Clinical Committee. Society for Endocrinology Endocrine Emergency Guidance: emergency management of acute hypercalcaemia in adult patients. Endocr Connect 2016;5:G9-11. 